\title{
Treatment of non-inflamed obstructive meibomian gland dysfunction by an infrared warm compression device
}

\author{
E Goto, Y Monden, Y Takano, A Mori, S Shimmura, J Shimazaki, K Tsubota
}

Br J Ophthalmol 2002;86:1403-1407

See end of article for authors' affiliations

.....................

Correspondence to: Eiki Goto, MD, Department of Ophthalmology, Tokyo Dental College, Sugano 5-11-13, Ichikawa-shi, Chiba 272-8513, Japan; eikigoto@nifty.com

Accepted for publication 2 July 2002

\begin{abstract}
Aim: To test the short term efficacy and safety of an infrared warm compression device (IWCD, Eye Hot, Cept Co, Tokyo, Japan) as treatment for non-inflamed meibomian gland dysfunction (MGD).

Methods: 37 subjects with non-inflamed obstructive MGD, with and without aqueous tear deficiency (ATD) dry eye, participated in a prospective non-comparative interventional case series. Symptom scores, face scores, tear evaporation rates, fluorescein and rose bengal vital staining, tear break up time (BUT), Schirmer test, meibomian gland obstruction, and meibography were compared before and after 2 weeks of therapy.

Results: In a total of 37 cases, total subjective symptom scores and subjective face scores improved significantly, from 12.3 (SD 5.9) to 8.4 (6.1), and from 7.0 (1.7) to 5.3 (2.0) (both $p<0.0001$ ). The results for tear evaporation rates during forced blinking $(p=0.002)$, fluorescein staining $(p=0.03)$, rose bengal staining $(p=0.03), B U T(p<0.0001)$, and meibomian gland orifice obstruction score ( $p$ $<0.0001$ ) had also improved significantly at the end of the 2 week period of infrared thermotherapy. No complaints and/or complications of the IWCD were reported.

Conclusion: The IWCD was effective and safe for the treatment of MGD. Improved tear stability associated with release of meibum is a possible mechanism of this treatment.
\end{abstract}

$\mathrm{T}$ he meibomian glands secrete lipid into tears, and the lipid forms the oily layer of the preocular tear film. This layer is responsible for preventing excessive evaporation of tears, for the barrier function at the lid margin, for lubrication during blinking, and for maintaining a smooth optical surface. ${ }^{1}$

Non-inflamed obstructive meibomian gland dysfunction (MGD) is the major cause of lipid tear deficiency (LTD) or evaporative dry eye, and has recently attracted attention as a cause of ocular discomfort. ${ }^{23}$ MGD decreases the supply of normal lipid, which leads to increased tear evaporation, decreased tear stability, loss of lubrication, and damage to the ocular surface epithelium, resulting in symptoms. ${ }^{45}$

The conventional treatment for MGD and blepharitis includes warm compression, lid hygiene, and topical or systemic medications. ${ }^{6-9}$ However, as the therapeutic efficacy of conventional warm compression is variable and this treatment must be continued over a long period of time to achieve satisfactory reduction of symptoms, patients often discontinue treatment. Therefore, a more convenient method of performing warm compression is necessary to improve patient compliance. We have preliminarily reported that an infrared warm compression device (IWCD, Eye Hot, Cept Co, Tokyo, Japan) was a safe and effective device for warming the eyelids in normal volunteers, and that it allows subjects to perform warm compression without any preparation by simply connecting the device to a power supply. ${ }^{10}$ In this study, we assessed the short term clinical efficacy and safety of the IWCD for the treatment of MGD.

\section{MATERIALS AND METHODS}

\section{Subjects and inclusion criteria for MGD}

We examined a series of 37 consecutive subjects with MGD (seven males, mean age 51.0 (SD 22.2) years, and 30 females, mean age 50.9 (13.3) years) at Tokyo Dental College, Ichikawa General Hospital.

These MGD subjects had already tried the conventional treatment for MGD in their follow up, such as the lid hygiene programme with warm compression with or without topical antibiotics and/or steroids, and/or oral tetracycline. MGD subjects with no satisfactory relief of ocular symptoms, such as dry eye sensation, ocular fatigue, ocular pain, foreign body sensation, irritation, burning sensation or itchiness with those treatments, participated in this study. After the conventional treatment had been found to be ineffective in improving symptoms, the subjects had been followed up with the preservative-free artificial tears (Soft Santear, Santen Pharmaceutical, Osaka, Japan). Seven of 37 subjects (19\%) dropped out of the lid hygiene programme with conventional warm compression.

Criteria for the diagnosis of non-inflamed obstructive meibomian gland dysfunction included the presence of meibomian gland dropout by meibography via transillumination through the tarsus, no or poor meibum expression by digital compression, and no or negligible inflammation in the lid margin. $^{3411}$ Transillumination examination (meibography) with a fibreoptic device (L-3920, Inami, Co, Tokyo, Japan) was performed, ${ }^{12}$ and the degree of meibomian gland dropout was scored as described previously: grade 0 , no gland dropout; grade 1, gland dropout in less than half of the inferior tarsus; and grade 2, gland dropout in more than half of the inferior tarsus. $^{411}$

To assess meibum expression and meibomian gland orifice obstruction, digital pressure was applied to the upper tarsus, and the degree of ease of expression of meibomian secretions (meibum) was evaluated semiquantitatively as follows: grade 0 , clear meibum easily expressed; grade 1, cloudy meibum expressed with mild pressure; grade 2, cloudy meibum expressed with more than moderate pressure; and grade 3 , meibum cannot be expressed even with strong pressure. ${ }^{41}$ Patients with a meibography score of grade 1 or 2 and a meibomian gland orifice obstruction score of 2 or 3 were diagnosed as having MGD.

Five of the subjects had Sjögren's syndrome and eight had a previous history of allergic conjunctivitis (but no active inflammation or itchiness during the study period). ${ }^{13}$ There were 17 subjects with a Schirmer test value of less than $5 \mathrm{~mm}$, indicating that they had non-inflamed obstructive MGD complicated by aqueous tear deficiency (ATD), ${ }^{14}$ while 20 patients 
Table 1 Temperature change of the cornea and upper eyelid in Japanese hot baths and IWCD use (mean (SD))

\begin{tabular}{|c|c|c|c|}
\hline & & $\begin{array}{l}\text { Cornea } \\
\left({ }^{\circ} \mathrm{C}\right)\end{array}$ & $\begin{array}{l}\text { Upper eyelid } \\
\left({ }^{\circ} \mathrm{C}\right)\end{array}$ \\
\hline \multirow[t]{3}{*}{ Normal volunteers $(n=5)$} & Room temperature $\left(22.5^{\circ} \mathrm{C}\right)$ & $34.8(0.9)$ & $35.0(0.7)$ \\
\hline & $\begin{array}{l}\text { In Japanese hot bath } 5 \text { minutes (water temperature, } \\
41.5^{\circ} \mathrm{C} \text {, bathroom temperature } 26.4^{\circ} \mathrm{C} \text { ) }\end{array}$ & $38.4(0.3)$ & $38.2(0.5)$ \\
\hline & IWCD 5 minutes & $37.9(0.9)$ & $38.8(0.7)$ \\
\hline \multirow[t]{2}{*}{ MGD subjects $(n=3)$} & Room temperature $\left(22.5^{\circ} \mathrm{C}\right)$ & $35.6(0.8)$ & $35.8(0.7)$ \\
\hline & IWCD 5 minutes & $38.2(1.7)$ & $37.4(0.9)$ \\
\hline \multirow[t]{2}{*}{ 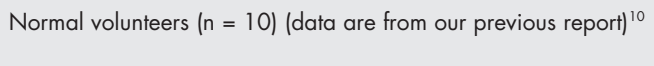 } & Room temperature $\left(23.0^{\circ} \mathrm{C}\right)$ & $34.1(1.2)$ & $34.1(0.8)$ \\
\hline & IWCD 5 minutes & $36.1(1.4)$ & $37.3(1.2)$ \\
\hline
\end{tabular}

IWCD = infrared warm compression device.

The temperature of the eye was measured using an infrared radiation thermometer (THI-500S, Tasco Japan, Osaka, Japan) ${ }^{26}$ for 5 normal volunteers and 3 MGD subjects, and also compared to the data from our previous report. ${ }^{10}$ The typical water temperature of Japanese hot baths (usual home bath, not a hot spring) is around $42^{\circ} \mathrm{C}$. The temperature of the cornea and upper eyelid were almost identical in both the Japanese hot baths and while using the IWCD, so the temperature change for 5 minutes of use of the IWCD was within the range of normal daily activities.

had Schirmer test values equal to or greater than $6 \mathrm{~mm}$. Analysis was done among all 37 MGD subjects, and MGD patients with and without ATD, separately. Only the right eye was used for analysis. Eyes with anterior blepharitis of moderate or greater severity, infectious conjunctivitis, eyes with an occluded punctum, and contact lens wearers were excluded from the study. Also, subjects with best corrected visual acuity less than 1.0 and obvious eyelid skin abnormalities, such as atopic dermatitis, were excluded. Current topical medication (preservative-free artificial tear eye drops) was continued unchanged throughout the study. Informed consent was obtained from all subjects, and institutional review board/ethnics committee approval was not required for this study.

\section{Assessment of symptoms, tears, ocular surface, and meibomian glands}

After a general ocular examination, the following examinations were carried out in the order listed to prevent the procedures from affecting each other: subjective symptoms, subjective face scores, tear evaporation test, fluorescein and rose bengal vital staining, measurement of BUT, Schirmer test, assessment of MG orifice obstruction, and meibography. Best corrected visual acuity was more than 1.0 in decimal notation in all subjects, and lens opacities were recorded according to the method of Taylor and West ${ }^{15}{ }^{16}:$ (1) nuclear opacity from grade 0 to 4 ; (2) cortex opacity; and (3) posterior subcapsular opacity.

Subjective symptoms were checked on dry eye sensation, ocular fatigue, ocular pain, foreign body sensation, irritation, burning sensation, and itchiness. All these seven symptoms were scored from 0 to 4 as follows: 0 , none; 1, mild 2; moderate, 3; moderate to severe; and 4, severe. Summation of these scores was used as a total subjective symptom score, thus minimum of summed score was 0 , and maximum was 28 .

A questionnaire on overall comfort was conducted by using a face scorecard, which was used in our previous report, ${ }^{10}$ showing nine faces, each with a different expression. ${ }^{17-19}$ For example, the saddest face (scored 9) describes severe discomfort, and the happiest face (scored 1) represents no discomfort.

Tear evaporation during normal blinking and forced blinking (subjects were asked to blink every 5 seconds while measuring tear evaporation) was measured by the method previously reported. ${ }^{20}$ The system is composed of a closed chamber with a small sensor for humidity and temperature, and goggles, which fitted tightly around the subjects' eyes. The data were recorded on a computer and the evaporation rate at $40 \%$ ambient humidity was calculated as a representative value. Blink rate was counted at tear evaporation measurement in normal blinking. Because the blinking rate was variable among subjects, we thought this rate might interfere with the tear evaporation results. Therefore, we measured the evaporation under both normal and forced blinking.

The ocular surface was examined by the double staining method. ${ }^{21}$ A $2 \mu \mathrm{l}$ volume of preservative-free solution consisting of $1 \%$ fluorescein and $1 \%$ rose bengal dye was applied to the conjunctival sac. The intensity of the rose bengal staining of the cornea and conjunctiva on the exposure zone was recorded, with a possible minimum score being 0 and a possible maximum score being 3 on each nasal conjunctiva, cornea, and temporal conjunctiva. ${ }^{22}$ Fluorescein staining of the cornea was also rated, with a possible minimum score being 0 and a possible maximum score being 3 on each upper, central, and lower cornea. ${ }^{1721}$ BUT was measured three times, and the measurements were averaged. ${ }^{21}$ Assessment of meibum expression, meibomian gland orifice obstruction, and gland dropout are described above in the inclusion criteria section. ${ }^{41}$ Schirmer test was performed to measure tear secretion without anaesthesia as proposed in the Japanese criteria for dry eye. ${ }^{23}{ }^{24}$ Examinations were performed before treatment and after 2 weeks of treatment.

\section{Device for warm compression}

The IWCD was adopted for convenient warm compression therapy..$^{10}$ Briefly, the IWCD is composed of two hard eye patches that are attached to the eye mask according to the pupillary distance of the subject. The eye mask is held in place by an elastic headband. Each patch has 19 light emitting diodes, emitting near infrared radiation from 850 to $1050 \mathrm{~nm}$, with a peak at $940 \mathrm{~nm}$. It produces approximately $10 \mathrm{~mW} / \mathrm{cm}^{2}$ of energy, operating on electrical power. Previously, we reported the safety and efficacy of the IWCD on 10 normal subjects using non-contact infrared radiation thermography and the subjects' comfort with the face score. ${ }^{10}$ In this study, the device was applied to all 37 MGD subjects for 5 minutes, after 2 minutes of preheating, twice a day for 2 weeks, with the eyelids closed.

\section{Temperature change of the eye using the device}

When we use near infrared radiation for ocular surface treatment with closed eyelids, thermal cataract formation could be a concern. ${ }^{25}$ To assess the temperature change of the cornea and eyelids during normal daily activities and while using the IWCD (wave length peak, $940 \mathrm{~nm}$ ), we measured the temperature of the eye using an infrared radiation thermometer (THI-500S, Tasco Japan, Osaka, Japan) ${ }^{26}$ among five normal volunteers (four females and one male, 28.0 (0.7) years of age) at room temperature, in Japanese hot baths (water temperature around $42^{\circ} \mathrm{C}$ ), and while using the IWCD (Table 1). Also the temperature of the eye was checked for three MGD subjects (two females and one male, 48.2 (17.4) years of age) at room temperature and while using the IWCD. The temperature of the cornea and upper eyelid were nearly identical both 


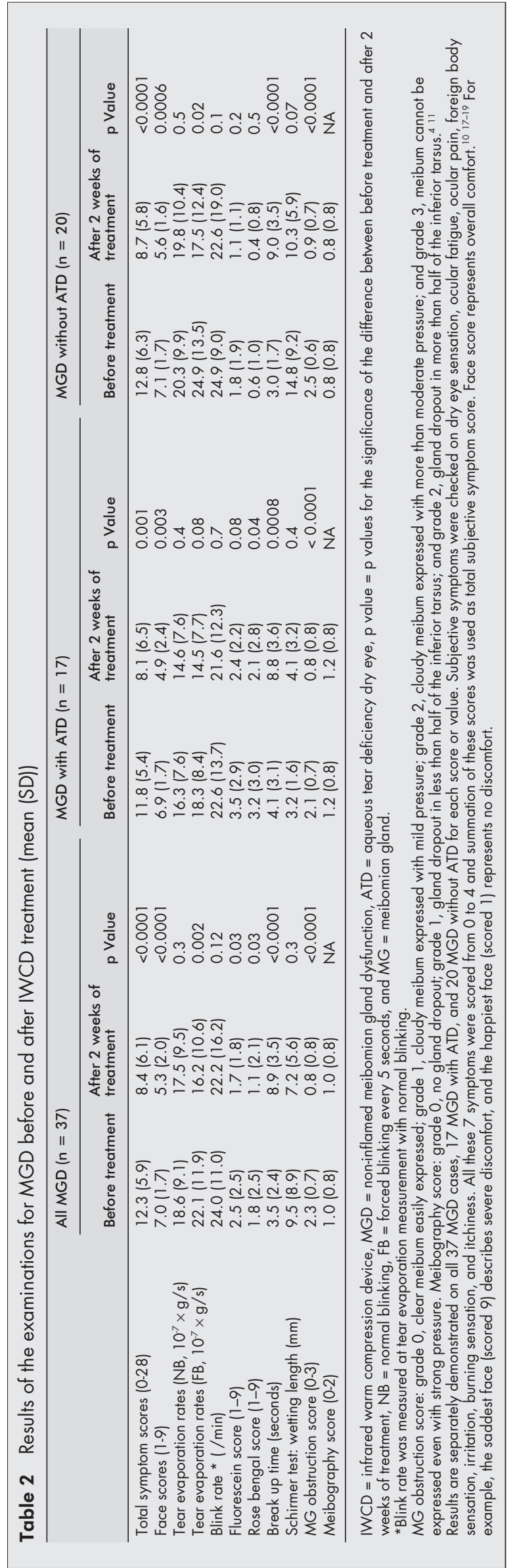

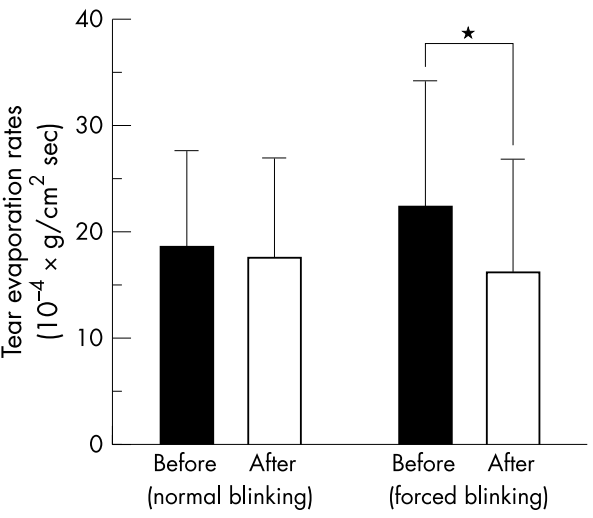

Figure 1 Results of tear evaporation rates during natural blinking and forced blinking in 37 cases with MGD. The difference in tear evaporation rates during normal blinking after 2 weeks of therapy was not significant, but the difference in evaporation during forced blinking was significant ( ${ }^{*} p=0.002$ ) in all 37 cases with MGD.

in the Japanese hot bath and while using the IWCD. Thus, we considered that a temperature change for 5 minutes of use of IWCD was within the range of normal daily activities.

\section{Study design and statistical analysis}

A prospective non-comparative interventional case series study was performed. All data are presented as means (SD). Symptoms and findings before and after 2 weeks of therapy were compared (Table 2 and Fig 1 ) in all subjects, and also in MGD subjects with and without ATD, separately. Data were analysed by Wilcoxon matched pairs signed ranks test for non-parametric paired data. A p level of $<0.05$ was accepted as statistically significant. GraphPad InStat 3 for Mac OS $\mathrm{X}$ (GraphPad Software, Inc, San Diego, CA, USA) was used for the statistical analysis.

\section{RESULTS}

The results of this study are summarised in Table 2. In a total of 37 cases, each scored symptom significantly improved except for the burning sensation (dry eye sensation from 2.6 (1.5) to $1.9(1.4), p=0.03$, ocular fatigue from $2.9(1.2)$ to 1.9 (1.2), $\mathrm{p}<0.0001$, ocular pain from 1.9 (1.5) to $1.3(1.3), \mathrm{p}=$ 0.002 , foreign body sensation from 1.6 (1.5) to $1.0(1.2), \mathrm{p}=$ 0.01 , irritation from 2.1 (1.7) to 1.5 (1.4), $\mathrm{p}=0.03$, burning sensation from $0.6(1.2)$ to $0.4(0.9), \mathrm{p}=0.1$, and itchiness from $0.7(0.7)$ to $0.5(0.7), p=0.02$, respectively). Total subjective symptom scores and subjective face scores improved significantly after 2 weeks of therapy. Tear evaporation rates during normal blinking did not change significantly after treatment ( $p=0.3$, Fig 1 ), but evaporation during forced blinking decreased significantly, from 22.1 (11.9) $\left(10^{-7} \mathrm{~g} / \mathrm{s}\right)$ to $16.2(10.6)\left(10^{-7} \mathrm{~g} / \mathrm{s}, \mathrm{p}=0.002\right.$, Fig $\left.\mathrm{l}\right)$. Vital staining score improved significantly (fluorescein score from 2.5 (2.5) to 1.7 (l.8), $\mathrm{p}=0.03$ and rose Bengal score from 1.8 (2.5) to 1.1 (2.1), $\mathrm{p}=0.03$, respectively). BUT was also prolonged significantly. The Schirmer test values and meibography scores did not change significantly. The meibomian gland orifice obstruction score improved, from $2.3(0.7)$ to $0.8(0.8)$ $(\mathrm{p}<0.0001$, Fig $2 \mathrm{~A}$ and B $)$.

When the results were compared in MGD cases with and without ATD separately (Table 2), in 17 MGD cases with ATD, total subjective symptom scores and subjective face scores also improved significantly. Tear evaporation rates during both normal blinking and forced blinking did not show significant change after treatment. Rose bengal staining score showed significant improvement from $3.2(3.0)$ to $2.1(2.8)(\mathrm{p}=0.04)$. On the contrary, fluorescein staining scores did not significantly change. BUT improved significantly. The meibomian 

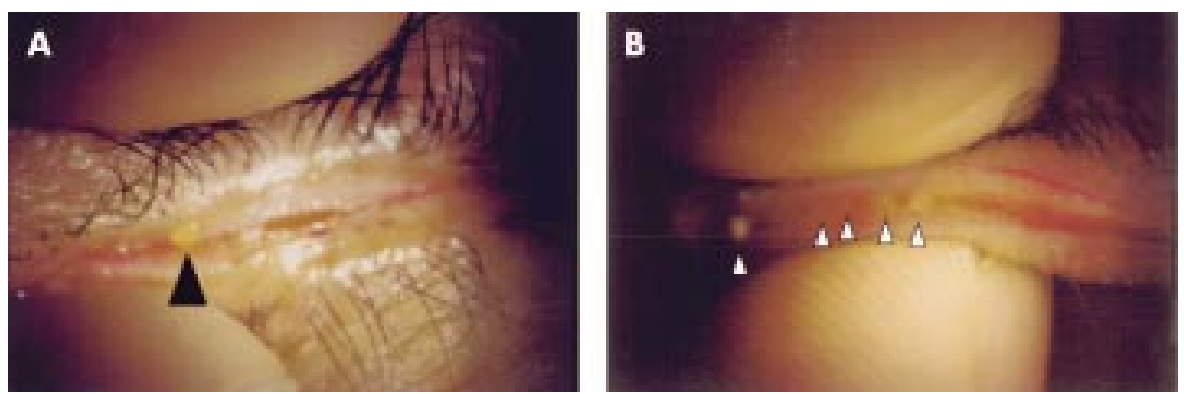

Figure 2 Meibum expression before (A) and after (B) infrared warm compression. (A) Hardly any meibum was expressed with obstructed meibomian orifice when digital pressure was applied before therapy (arrowhead, interpalpebral red dye is rose bengal dye, obstruction score was 3). (B) After the therapy, the solid meibum was melted by the temperature of the device and was relatively easily expressed by digital pressure (white arrowheads, obstruction score was 1).

gland orifice obstruction scores improved, from 2.1 (0.7) to 0.8 (0.8) $(\mathrm{p}<0.0001)$.

Similarly, in 20 MGD cases without ATD, total subjective symptom scores and subjective face scores improved. Tear evaporation rates during normal blinking did not change significantly after treatment, but evaporation during forced blinking decreased significantly, from 24.9 (13.5) $\left(10^{-7} \mathrm{~g} / \mathrm{s}\right)$ to $17.5(12.4)\left(10^{-7} \mathrm{~g} / \mathrm{s}, \mathrm{p}=0.02\right)$. Fluorescein and rose bengal staining score did not show significant change. On the other hand, BUT improved significantly. The meibomian gland orifice obstruction score improved, from 2.5 (0.6) to 0.9 (0.7) $(\mathrm{p}<0.0001)$.

No complaints attributable to the IWCD were reported. No complications, such as lid skin dermatitis, anterior chamber inflammation, decreased visual acuity, or cataract formation were observed over the 2 week duration of this study.

\section{DISCUSSION}

This study demonstrated a clear benefit of using the IWCD to treat MGD. Since MGD is a major cause of ocular irritation, relief of symptoms is an essential goal of treatment. All measured symptoms (dry eye sensation, ocular fatigue, ocular pain, foreign body sensation, irritation, and itchiness) except burning sensation showed significant improvement. Also, ocular discomfort as checked by the subjective face score decreased.

Objective findings, including tear evaporation, BUT, fluorescein staining, rose bengal staining, and MG orifice obstruction improved in all 37 cases. No subjective complaints or objective complications as a result of IWCD use were noted after 2 weeks of therapy with the IWCD, indicating that it is a safe device, and all subjects continued to use it for 2 weeks with no dropout during the treatment period. Since the use of the IWCD is more convenient than conventional warm compression, we were able to achieve good compliance with this therapy. In addition, the IWCD is portable and can be used wherever electricity is available, such as in the office after visual display terminal work, ${ }^{27}$ even if the subject worked in an active office.

Tear stability, tear evaporation, and meibum expression all improved. The improved tear evaporation during forced blinking, which was observed in the comparison in all 37 cases as well as in the 20 cases of MGD without ATD, suggests that meibum was released by infrared heating, spread well to form a stable lipid layer, and decreased the evaporation. ${ }^{28}{ }^{29}$ No significant change of tear evaporation was observed in 20 MGD with ATD subjects. This might suggest that subjects without a proper aqueous amount could not form the lipid film to decrease evaporation even though they had improved lipid expression. ${ }^{30}$ Tear evaporation rates measured during forced blinking every 5 seconds may be a more sensitive means of detecting the abnormal tear lipid layer of MGD. MGD patients may compensate for dryness and increased tear evaporation by frequent blinking in the measurement of tear evaporation with normal blinking. In this study the blink rate measured during the evaporation examination with normal blinking was $24.0(11.0) / \mathrm{min}$ in all $37 \mathrm{MGD}$ patients, while the blink rate was reported as $14.3 / \mathrm{min}$ in normal subjects and 33.9/min in dry eye patients. ${ }^{31}{ }^{32}$ Although not performed in this study, adding lid massage to warm compression could suppress the evaporation even more during the evaporation measurement with normal blinking. Tear evaporation rates were used for the first time to evaluate the efficacy of treating MGD. To directly assess the change in the tear lipid layer on the cornea after the treatment, further development of tear interference image for quantification of the lipid layer in MGD is expected. ${ }^{60}$

The mechanism of the decrease in meibomian gland orifice obstruction using IWCD is unclear. McCulley et al found that meibomian secretions with ester fractions of different composition can have different melting points, and that chronic blepharitis can cause a shift towards lipid with higher melting points, producing a stagnant, and less dynamic, tear film..$^{33-36}$ Heating the eye and eyelids with the IWCD is speculated to melt the obstructed meibomian gland lipid easily, similar to the previous warm compression, as infrared has been used for effective hyperthermia. ${ }^{10}$ This may lead to the improvement of tear stability indicated by BUT. Also, this infrared heater was reported to have the advantage of warming the eyelid effectively and safely with its strong emission at $940 \mathrm{~nm}$, compared to the traditional Asian eye warming instrument using the wide range (200-6000 nm) electric light bulb. ${ }^{10}$ Infrared between 800 and $1200 \mathrm{~nm}$ easily passes through skin and is mostly absorbed in the underlying $1-3 \mathrm{~mm}$ of tissue. ${ }^{37} 38$

It has been considered that an unstable tear film in MGD patients leads to epithelial damage as shown by fluorescein and rose bengal vital staining. ${ }^{39}$ IWCD treatment was also effective in reducing the damage to the cornea and conjunctival epithelium, in all 37 cases. However, these effects were limited when we analysed the results separately between MGD with and without ATD, showing significant improvement only in the rose bengal scores of MGD subjects with ATD.

No significant changes in the Schirmer test values or meibography scores were reported after 2 weeks of treatment. Thus, the IWCD did not change the tear secretion from the lacrimal gland and visible structure of meibomian gland of MGD patients. ${ }^{40}$

Although the safety of the IWCD with near infrared radiation with the eyelids closed has been described previously, ${ }^{10}$ and although infrared radiation has been used for transpupillary thermotherapy, ${ }^{41}{ }^{42}$ near infrared radiation could cause damage to the eye, especially thermal cataract formation. ${ }^{25}{ }^{43-45}$ Thus, the temperature change in use of the IWCD is important. In this study, we compared the temperature change in both 5 minutes of use of the IWCD and 5 minutes of taking a Japanese hot bath, and the change was found to be identical. Although a long term study for the safety of the device would be required, we considered that the device was 
safe for at least 2 weeks of use, as the range of temperature change was within normal daily activities. As for the skin, the first step of thermal injury to human skin occurs in temperatures under $44^{\circ} \mathrm{C}$ of the surface temperature of the skin. ${ }^{46}$ Since eyelid temperature after IWCD application was never beyond $39.7^{\circ} \mathrm{C}$, it was unlikely to cause thermal injury to the skin by using the IWCD. ${ }^{47}$

Application of the treatment in this study was limited to non-inflamed MGD with and without ATD. We have no experience with this treatment for MGD with active inflammation, such as meibomitis and blepharitis, and conventional therapy ${ }^{9}$ should be tried first for these diseases. Also, if we use the conventional medication, such as topical antibiotics, with IWCD for the treatment of MGD, it could be more effective.

In summary, we found that the IWCD was effective and safe in the treatment of MGD after 2 weeks of therapy. Improvement of tear stability associated with the release of meibum was considered to be responsible for its therapeutic effect.

\section{ACKNOWLEDGEMENTS}

Supported by grants from the Japanese Ministry of Education and Science, Tokyo, the Medical School Faculty and Alumni Grants of Keio University Medical School, Tokyo, and the Hightech Research Center at Tokyo Dental College, Chiba, Japan. The authors thank Cept Co, Tokyo, Japan, for its assistance in developing the infrared warm compression device, "Eye Hot."

Financial disclosure: Patent pending for infrared warm compression device by Kazuo Tsubota, MD.

\section{Authors' affiliations}

E Goto, Y Takano, S Shimmura, J Shimazaki, K Tsubota, Tokyo

Dental College, Department of Ophthalmology, Chiba, Japan

E Goto, Y Takano, A Mori, S Shimmura, K Tsubota, Keio University, Department of Ophthalmology, Tokyo, Japan

Y Monden, Kurume University, Department of Ophthalmology, Kurume, Japan

\section{REFERENCES}

1 Tiffany JM. The lipid secretion of the meibomian glands. Adv Lipid Res 1987;22:1-62

2 Lemp MA. Report of the National Eye Institute/Industry workshop on clinical trials in dry eyes. CLAO J 1995;21:221-32.

3 Lee SH, Tseng SC. Rose bengal staining and cytologic characteristics associated with lipid tear deficiency. Am J Ophthalmol 1997; 124:736-50.

4 Shimazaki J, Sakata M, Tsubota K. Ocular surface changes and discomfort in patients with meibomian gland dysfunction. Arch Ophthalmol 1995;113:1266-70.

5 Mathers WD. Ocular evaporation in meibomian gland dysfunction and dry eye. Ophthalmology 1993;100:347-51.

6 Korb DR, Greiner JV. Increase in tear film lipid layer thickness following treatment of meibomian gland dysfunction. Adv Exp Med Biol 1994;350:293-8.

7 Key JE. A comparative study of eyelid cleaning regimens in chronic blepharitis. CLAO J 1996;22:209-12.

8 Smith RE, Flowers CW Jr. Chronic blepharitis: a review. CLAO J 1995;21:200-7.

9 Driver PJ, Lemp MA. Meibomian gland dysfunction. Surv Ophthalmol 1996;40:343-67.

10 Mori A, Oguchi Y, Goto E, et al. Efficacy and safety of infrared warming of the eyelids. Cornea 1999;18:188-93.

11 Shimazaki J, Goto $E$, Ono $M$, et al. Meibomian gland dysfunction in patients with Siögren syndrome. Ophthalmology 1998;105: 1485-8.

12 Robin JB, Jester JV, Nobe J, et al. In vivo transillumination biomicroscopy and photography of meibomian gland dysfunction. A clinical study. Ophthalmology 1985:92:1423-6.

13 Fox RI, Robinson CA, Curd JG, et al. Sjögren's syndrome. Proposed criteria for classification. Arthritis Rheum 1986;29:577-85.

14 Xu KP, Yagi Y, Toda I, et al. Tear function index. A new measure of dry eye. Arch Ophthalmol 1995;113:84-8.
15 Taylor HR, West SK. The clinical grading of lens opacities. Aust NZ J Ophthalmol 1989;17:81-6.

16 Brown NAP, Bron AJ. Lens disorders: a clinical manual of cataract diagnosis. 1st ed. Oxford: Butterworth-Heinemann, 1996.

17 Tsubota K, Goto E, Fujita H, et al. Treatment of dry eye by autologous serum application in Siögren's syndrome. Br J Ophthalmol 1999;83:390-5

18 Goto E, Shimmura S, Shimazaki J, et al. Treatment of superior limbic keratoconjunctivitis by application of autologous serum. Cornea 2001;20:807-10.

19 Tsubota K, Monden Y, Yagi Y, et al. New treatment of dry eye: the effect of calcium ointment through eyelid skin delivery. Br J Ophthalmol 1999;83:767-70

20 Tsubota K, Yamada M. Tear evaporation from the ocular surface. Invest Ophthalmol Vis Sci 1992:33:2942-50.

21 Toda I, Tsubota K. Practical double vital staining for ocular surface evaluation (letter). Cornea 1993;12:366-7

22 Van Bijsterveld OP. Diagnostic tests in the sicca syndrome. Arch Ophthalmol 1969;82:10-14

23 Schirmer $\mathbf{O}$. Studien zur physiologie und pathologie der tranenabsonderung und tranenabfuhr. Albrebt von Graefes Archiv fur Ophthalmologie 1903;56:197-291.

24 Shimazaki J. Definition and criteria of dry eye. Ganka [Ophthalmol, Jap] 1995;37:765-70.

25 Okuno T. Thermal effect of visible light and infra-red radiation (i.r.-A, i.r.-B and i.r. C) on the eye: a study of infra-red cataract based on a model. Ann Occup Hyg 1994;38:351-9.

26 Fujishima H, Toda I, Yamada M, et al. Corneal temperature in patients with dry eye evaluated by infrared radiation thermometry. $\mathrm{Br} J$ Ophthalmol 1996;80:29-32.

27 Tsubota K, Nakamori K. Dry eyes and video display terminals (letter). N Engl J Med 1993;328:584

28 Mishima S, Maurice DM. The oily layer of the tear film and evaporation from the corneal surface. Exp eye Res 1961;1:39-45.

29 Tiffany JM, Winter N, Bliss G. Tear film stability and tear surface tension. Curr Eye Res 1989;8:507-15.

30 Yokoi N, Mossa F, Tiffany JM, et al. Assessment of meibomian gland function in dry eye using meibometry. Arch Ophthalmol 1999; 117:723-9.

31 Tsubota K, Hata S, Okusawa Y, et al. Quantitative videographic analysis of blinking in normal subjects and patients with dry eye. Arch Ophthalmol 1996;1 14:715-20.

32 Goto E, Yagi Y, Matsumoto Y, et al. Impaired functional visual acuity of dry eye patients. Am J Ophthalmol 2002;133:181-6.

33 Dougherty JM, McCulley JP. Analysis of the free fatty acid component of meibomian secretions in chronic blepharitis. Invest Ophthalmol Vis Sci 1986;27:52-6

34 McCulley JP, Dougherty JM, Deneau DG. Classification of chronic blepharitis. Ophthalmology 1982;89:1173-80.

35 Osgood JK, Dougherty JM, McCulley JP. The role of wax and sterol esters of meibomian secretions in chronic blepharitis. Invest Ophthalmol Vis Sci 1989;30:1958-61.

36 Shine WE, McCulley JP. Keratoconjunctivitis sicca associated with meibomian secretion polar lipid abnormality. Arch Ophthalmol 1998; 1 16:849-52.

37 Palmer KF, Williams D. Optical properties of water in near-infrared. J Opt Soc Am 1974;64:1 107-10.

38 Yoshii K, Morita M. Effect on a living body of infrared radiation heating apparatus. J Illum Engng Inst Jpn 1990;74:26-9.

39 Feenstra RP, Tseng SC. Comparison of fluorescein and rose bengal staining. Ophthalmology 1992;99:605-17.

40 Gutgesell VJ, Stern GA, Hood Cl. Histopathology of meibomian gland dysfunction. Am J Ophthalmol 1982;94:383-7.

41 Garcia-Arumi J, Ramsay LS, Guraya BC. Transpupillary thermotherapy for circumscribed choroidal hemangiomas. Ophthalmology 2000; 107:351-6; discussion 357

42 Robertson DM, Buettner H, Bennett SR. Transpupillary thermotherapy as primary treatment for small choroidal melanomas. Arch Ophthalmol 1999:117:1512-9.

43 Okuno T. Thermal effect of infra-red radiation on the eye: a study based on a model. Ann Occup Hyg 1991;35:1-12.

44 Wolbarsht M. Damage to the lens from infrared. Proc Soc Photo-optic Inst Engrs 1980;229:121-42.

45 Lydahl E, Philipson B. Infrared radiation and cataract. I. Epidemiologic invevestigation of iron- and steel-workers. Acta Ophthalmol 1984;62:961-75

46 Moritz AR, Henriques FC. Studies of thermal injury II. The relative importance of time and surface temperature in the causation of cutaneous burns. Am. J. Pathol 1947;23:695-720.

47 Steinhausen D, Mayer WK, von Ardenne M. Evaluation of systemic tolerance of 42.0 degrees $C$ infrared-A whole-body hyperthermia in combination with hyperglycemia and hyperoxemia. A Phase-l study. Strahlenther Onkol 1994;170:322-34. 